\title{
THE TANGENT BUNDLE OF THE LONG LINE
}

JAMES A. MORROW

We prove the following surprise.

THEOREM. The tangent bundle of the long line is not trivial.

Proof. The long line $L$ is defined in Hocking and Young [1], for example. It is easy to check that $L$, with the topology defined there, supports the structure of a $C^{\infty}$ manifold. In fact, it is proved in [2] that $L$ (there called the Alexandroff Half Line) can be made into a real analytic manifold (I thank the referee for this reference). Let us recall what it means for a real vector bundle $E$ to be trivial. The bundle $E \stackrel{\pi}{\rightarrow} X$ is trivial if there is a bundle map $\phi: E \rightarrow \xi^{n}$ covering the identity: $X \rightarrow X$, where $\xi^{n}$ is the trivial $R^{n}$ bundle over $X$ and $n$ is the dimension of the fibre of $E$. But this is easily seen to be equivalent to the existence of a bundle map $\tilde{\phi}: E \rightarrow R^{n}$ where $R^{n}$ is considered as a bundle over a point $*$. (If $\omega: \xi^{n} \rightarrow R^{n}$ is projection onto the coordinate of each fibre, then $\tilde{\phi}=\omega \circ \phi$ is such a map. If $\tilde{\phi}$ is given then $\tilde{\phi}^{-1} R^{n}$ $=\xi^{n}$ and $E \cong \tilde{\phi}^{-1} R^{n}$ where $\tilde{\phi}^{-1}$ means the pull back under $\tilde{\phi}$.) So let $T L$ be the tangent bundle of $L$ and $\phi$ be a $C^{\infty}$ trivialization

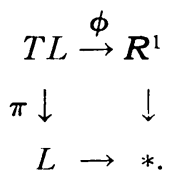

Then $g(x, y)=\phi(x) \cdot \phi(y)$ defines a $C^{\infty}$ positive definite inner product on the fibres of $T L$ ( $\phi$ is an isomorphism on each fibre). Thus $L$ would be a Riemannian manifold. But it is well known (see Kobayashi-Nomizu [3, p. 166]) that a Riemannian manifold (not necessarily assumed paracompact) has a metric space structure which defines the manifold topology. Now it is well known that $L$ is not paracompact, and hence is not a metric space. This contradiction proves the theorem.

\section{REFERENCES} 1961.

1. J. G. Hocking and G. S. Young, Topology, Addison-Wesley, Reading, Mass.,

2. H. Kneser, Analytische Struktur und Abzählbarkeit, Ann. Acad. Sci. Fenn. Ser. A.I. No. 251/5 (1968), 8 pp.

3. S. Kobayashi and K. Nomizu, Foundations of differential geometry. Vol. I, Interscience, New York, 1963.

University of California, Berkeley

Received by the editors January 24, 1969. 